\title{
NILPOTENT TOEPLITZ OPERATORS ON REINHARDT DOMAINS
}

\author{
MEHMET ÇELIK AND YUNUS E. ZEYTUNCU
}

ABSTRACT. We construct explicit examples of nontrivial nilpotent Toeplitz operators on Bergman spaces of certain Reinhardt domains in $\mathbb{C}^{2}$.

\section{Introduction.}

1.1. Set-up and results. Let $\Omega$ be a domain in $\mathbb{C}^{n}$ and $A^{2}(\Omega)$ denote the Bergman space of $\Omega$. The Bergman projection operator $\mathcal{B}_{\Omega}$ is the orthogonal projection from $L^{2}(\Omega)$ onto $A^{2}(\Omega)$. It is an integral operator with the kernel called the Bergman kernel, denoted by $B_{\Omega}(z, w)$. If $\left\{e_{n}(z)\right\}_{n=0}^{\infty}$ is an orthonormal basis for $A^{2}(\Omega)$, then the Bergman kernel can be represented as

$$
B_{\Omega}(z, w)=\sum_{n=0}^{\infty} e_{n}(z) \overline{e_{n}(w)} .
$$

See [11] for the general theory of Bergman spaces.

For a function $u$ on $\Omega$, the Toeplitz operator $T_{u}: A^{2}(\Omega) \rightarrow A^{2}(\Omega)$ with the symbol $u$ is defined by $T_{u}(f)=\mathcal{B}_{\Omega}(u f)$.

In this note, we are interested in the zero product problem. For two symbols $u_{1}$ and $u_{2}$, if the product $T_{u_{1}} T_{u_{2}}$ is identically zero on $A^{2}(\Omega)$, then can we claim $T_{u_{1}}$ or $T_{u_{2}}$ is identically zero? This is a non-trivial problem, and the answer is not even known when $\Omega$ is the unit disc.

Here, we indicate the problem has a different flavor in higher dimensions. In particular, we present a family of Reinhardt domains in $\mathbb{C}^{2}$

2010 AMS Mathematics subject classification. Primary 47B35, Secondary $32 \mathrm{~A} 36$.

Keywords and phrases. Toeplitz operator, Bergman space, nilpotent operator, Reinhardt domain.

Received by the editors on June 25, 2014, and in revised form on November 20, 2014. 
on which not only zero products but nilpotent Toeplitz operators of non-trivial Bergman space Toeplitz operators exist.

Theorem 1.1. There exist Reinhardt domains in $\mathbb{C}^{2}$ on whose Bergman spaces there are nilpotent Toeplitz operators.

Remark 1.2. It becomes clear in the proof that the operators in Theorem 1.1 are also of infinite rank.

1.2. History. The zero product problem on the Hardy space is initiated in [5]. It is completely solved in [3], where authors established that the product of non-zero Toeplitz operators is never zero. For the intermediate results, before the complete solution, see $[\mathbf{9}, \mathbf{1 0}]$ and the references in [3].

In [1], it is shown that, for the Toeplitz operators on the Bergman space $A^{2}(\mathbb{D})$ of the unit disc $\mathbb{D}$, the analogue of the Brown-Halmos theorem holds under an additional hypothesis that $u$ and $v$ are bounded and harmonic. Later, the same result is proven for radial symbols in [2]. The problem on $\mathbb{D}$, without extra assumptions on the symbols, remains open.

The higher-dimensional cases are studied in $[\mathbf{6}, \mathbf{7}, \mathbf{8}]$, where the results on the unit disc are extended to the ball or to the polydisk. In these papers, neither non-trivial zero products nor nilpotent Toeplitz operators are observed.

In [4], the problem is considered on the Segal-Bargmann space (the space of square integrable entire functions on $\mathbb{C}^{n}$ with a Gaussian decay weight) and an example of a non-trivial zero product of three Toeplitz operators is constructed. However, no nilpotent Toeplitz operator is observed.

2. Proof of Theorem 1.1. Inspired by the construction in [12], we define the following family of domains $\Omega_{m}$ in $\mathbb{C}^{2}$.

$$
\begin{aligned}
X & =\left\{\left(z_{1}, z_{2}\right) \in \mathbb{C}^{2}:\left|z_{1}\right|>e, \quad\left|z_{2}\right|<\frac{1}{\left|z_{1}\right| \log \left|z_{1}\right|}\right\}, \\
Y_{m} & =\left\{\left(z_{1}, z_{2}\right) \in \mathbb{C}^{2}:\left|z_{2}\right|>2, \quad|| z_{1}\left|-\frac{1}{\left|z_{2}\right|}\right|<\frac{1}{\left|z_{2}\right|^{m}}\right\},
\end{aligned}
$$




$$
Z=\left\{\left(z_{1}, z_{2}\right) \in \mathbb{C}^{2}:\left|z_{1}\right| \leq e, \quad\left|z_{2}\right| \leq 2\right\}
$$

and put

$$
\Omega_{m}=X \cup Y_{m} \cup Z, \quad m=1,2, \ldots
$$

Each $\Omega_{m}$ is an unbounded Reinhardt domain with finite volume, see Figure 1.

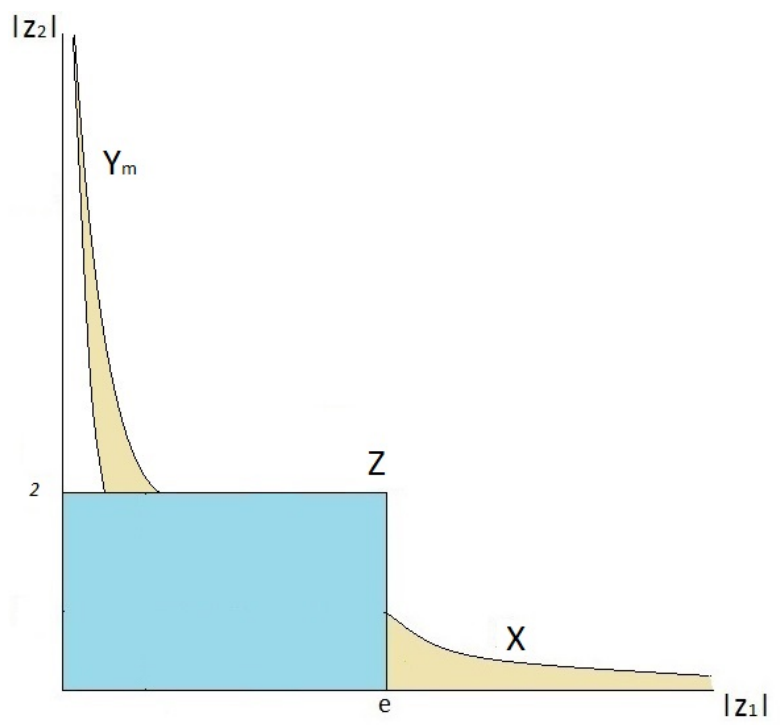

FIGURE 1. Representation of $\Omega_{m}$ in absolute space $\left\{\left(r_{1}, r_{2}\right) \in \mathbb{R}^{2} \mid r_{1} \geq 0\right.$ and $\left.r_{2} \geq 0\right\}$, under the map $\tau:\left(z_{1}, z_{2}\right) \rightarrow\left(\left|z_{1}\right|,\left|z_{2}\right|\right)$.

Lemma 2.1. For a multi-index $\alpha=\left(\alpha_{1}, \alpha_{2}\right)$, the monomial $z^{\alpha}$ is in $A^{2}\left(\Omega_{m}\right)$ if and only if $\alpha_{2} \geq \alpha_{1}>\alpha_{2}-(m-1) / 2$.

Proof. We begin with the calculation on the domain $X$.

$$
\begin{aligned}
\int_{X}\left|z^{\alpha}\right|^{2} d V(z) & =\int_{\left|z_{1}\right|>e}\left|z_{1}\right|^{2 \alpha_{1}} \int_{\left|z_{2}\right|<1 /\left|z_{1}\right| \log \left|z_{1}\right|}\left|z_{2}\right|^{2 \alpha_{2}} d A\left(z_{2}\right) d A\left(z_{1}\right) \\
& =4 \pi^{2} \int_{e}^{\infty} r_{1}^{2 \alpha_{1}+1} \int_{0}^{1 /\left(r_{1} \log \left(r_{1}\right)\right)} r_{2}^{2 \alpha_{2}+1} d r_{2} d r_{1}
\end{aligned}
$$




$$
=\frac{4 \pi^{2}}{2 \alpha_{2}+2} \int_{e}^{\infty} \frac{r_{1}^{2 \alpha_{1}+1}}{r_{1}^{2 \alpha_{2}+2}\left(\log \left(r_{1}\right)\right)^{2 \alpha_{2}+2}} d r_{1} .
$$

We note that, for $k>0$, the improper integral

$$
\int_{e}^{\infty} \frac{1}{x^{m}(\log x)^{k}} d x
$$

converges if and only if $m \geq 1$. Therefore, the last integral above (where $k=2 \alpha_{2}+2>0$ and $m=2\left(\alpha_{2}-\alpha_{1}\right)+1$ ) is finite if and only if $\left(\alpha_{2}-\alpha_{1}\right) \geq 0$. In other words,

$$
z^{\alpha} \in A^{2}(X) \Longleftrightarrow \alpha_{2} \geq \alpha_{1} \text {. }
$$

We continue with the calculation on domain $Y_{m}$.

$$
\begin{aligned}
\int_{Y_{m}}\left|z^{\alpha}\right|^{2} d V(z) & =\int_{\left|z_{2}\right|>2}\left|z_{2}\right|^{2 \alpha_{2}} \int_{\left(1 /\left|z_{2}\right|\right)-\left(1 /\left|z_{2}\right|^{m}\right)<\left|z_{1}\right|<\left(1 /\left|z_{2}\right|\right)+\left(1 /\left|z_{2}\right|^{m}\right)} \cdot \cdot\left|z_{1}\right|^{2 \alpha_{1}} d A\left(z_{2}\right) d A\left(z_{1}\right) \\
& =4 \pi^{2} \int_{2}^{\infty} r_{2}^{2 \alpha_{2}+1} \int_{1 / r_{2}-1 / r_{2}^{m}}^{1 / r_{2}+1 / r_{2}^{m}} r_{1}^{2 \alpha_{1}+1} d r_{1} d r_{2} \\
& =\frac{4 \pi^{2}}{2 \alpha_{1}+2} \int_{2}^{\infty} r_{2}^{2 \alpha_{2}+1}\left[\left(\frac{1}{r_{2}}-\frac{1}{r_{2}^{m}}\right)^{2 \alpha_{1}+2}\right. \\
& \left.-\left(\frac{1}{r_{2}}+\frac{1}{r_{2}^{m}}\right)^{2 \alpha_{1}+2}\right] d r_{2}
\end{aligned}
$$

Since $r_{2}>2$, after using the binomial expansion in the brackets, we consider the term $1 / r_{2}$ with the smallest degree as the dominant, which is $1 / r_{2}^{2 \alpha_{1}+1+m}$. The last integral can be estimated by:

$$
\int_{Y_{m}}\left|z^{\alpha}\right|^{2} d V(z) \approx \int_{2}^{\infty} r_{2}^{2 \alpha_{2}+1} \frac{1}{r_{2}^{2 \alpha_{1}+1+m}} d r_{2} .
$$

The integral on the right is finite if and only if $\alpha_{1}>\alpha_{2}+(1-m) / 2$. In other words,

$$
z^{\alpha} \in A^{2}\left(Y_{m}\right) \Longleftrightarrow \alpha_{1}>\alpha_{2}+\frac{1-m}{2} .
$$

Lemma 2.1 follows from equations (2.1) and (2.2).

Next, we set $m \geq 6, \phi=z_{1} / \bar{z}_{1}$ and consider $T_{\phi}$ on $A^{2}\left(\Omega_{m}\right)$. 
Proposition 2.2. The following properties hold:

(i) $T_{\phi}$ is not a zero operator.

(ii) $T_{\phi}$ does not have finite rank.

(iii) $T_{\phi}$ is a bounded operator.

(iv) $T_{\phi}$ is a nilpotent operator of degree $\lfloor m / 4\rfloor$, the largest integer less than or equal to $\mathrm{m} / 4$.

Remark 2.3. Once we prove Proposition 2.2, we immediately obtain Theorem 1.1. However, it will be clear in the proof that the domain and the operator we present are not unique but part of a family of domains and operators. We leave exploration of more examples to the reader.

Before starting the proof of Proposition 2.2, we define the following lattice for $m \geq 6$ :

$$
\begin{aligned}
R_{m} & =\left\{\left(\alpha_{1}, \alpha_{2}\right) \in \mathbb{N}^{2} \mid \alpha_{2} \geq \alpha_{1}>\alpha_{2}-\frac{m-1}{2}\right\} \\
& =\left\{\left(\alpha_{1}, \alpha_{2}\right) \in \mathbb{N}^{2} \mid \alpha_{1}+\frac{m-1}{2}>\alpha_{2} \geq \alpha_{1}\right\} .
\end{aligned}
$$

Remark 2.4. Shifting $\alpha_{1}$ to the right by a number $s$ greater than or equal to $(m-1) / 2$ is enough to put the resulting index $\left(\alpha_{1}+s, \alpha_{2}\right)$ out of $R_{m}$, that is, if $\left(\alpha_{1}, \alpha_{2}\right) \in R_{m}$, then for $s \geq(m-1) / 2$, we get $\left(\alpha_{1}+s, \alpha_{2}\right) \notin R_{m}$.

For a multi-index $\gamma=\left(\gamma_{1}, \ldots, \gamma_{n}\right) \in \mathbb{N}^{n}$, we set

$$
c_{\gamma}^{2}=\int_{\Omega}\left|z^{\gamma}\right|^{2} d V(z) .
$$

Then, on a radially symmetric domain $\Omega$ that contains the origin, the set (or a subset of) $\left\{z^{\gamma} / c_{\gamma}\right\}_{\gamma \in \mathbb{N}^{n}}$ gives a complete orthonormal basis for $A^{2}(\Omega)$. Each $f \in A^{2}(\Omega)$ can be written in the form

$$
f(z)=\sum_{\gamma} f_{\gamma} \frac{z^{\gamma}}{c_{\gamma}},
$$

where the sum converges in $A^{2}(\Omega)$, but also uniformly on compact subset of $\Omega$. For the coefficients $f_{\gamma}$, we have $f_{\gamma}=\left\langle f(z), z^{\gamma} / c_{\gamma}\right\rangle_{\Omega}$. 
Proof of Proposition 2.2. Consider $T_{\phi}$ on $A^{2}\left(\Omega_{m}\right)$ for $m \geq 6$. $\Omega_{m}$ is a radially symmetric domain and the monomials with exponents that reside in $R_{m}$ form a complete system for $A^{2}\left(\Omega_{m}\right)$. By using the orthogonality of monomials we obtain

$$
\begin{aligned}
T_{\phi}\left(z^{\alpha}\right)=\mathcal{B}_{\Omega_{m}}\left(\frac{z_{1}}{\bar{z}_{1}} \cdot z^{\alpha}\right) & =\sum_{\gamma \in R_{m}}\left\langle\frac{z_{1}}{\bar{z}_{1}} z^{\alpha}, \frac{z^{\gamma}}{c_{\gamma}}\right\rangle \frac{z^{\gamma}}{c_{\gamma}} \\
& =\frac{c_{\left(\alpha_{1}+1, \alpha_{2}\right)}^{2}}{c_{\left(\alpha_{1}+2, \alpha_{2}\right)}^{2}} z_{1}^{\alpha_{1}+2} z_{2}^{\alpha_{2}} .
\end{aligned}
$$

On the above summation only $\left(\gamma_{1}, \gamma_{2}\right)=\left(\alpha_{1}+2, \alpha_{2}\right)$ survives. Moreover, there exist multi-indices $\left(\alpha_{1}, \alpha_{2}\right)$ in $R_{m}$ such that $\left(\alpha_{1}+2, \alpha_{2}\right)$ is also in $R_{m}$. Therefore, there exists $z^{\alpha} \in A^{2}\left(\Omega_{m}\right)$ such that

$$
T_{\phi}\left(z^{\alpha}\right)=\frac{c_{\left(\alpha_{1}+1, \alpha_{2}\right)}^{2}}{c_{\left(\alpha_{1}+2, \alpha_{2}\right)}^{2}} z_{1}^{\alpha_{1}+2} z_{2}^{\alpha_{2}} \in A^{2}\left(\Omega_{m}\right)
$$

and $T_{\phi}$ is a non-zero operator.

For $m \geq 6$ and $k \in \mathbb{N}, z_{1}^{k} z_{2}^{k+2} \in A^{2}\left(\Omega_{m}\right)$ and

$$
T_{\phi}\left(z_{1}^{k} z_{2}^{k+2}\right)=\frac{c_{(k+1, k+2)}^{2}}{c_{(k+2, k+2)}^{2}} z_{1}^{k+2} z_{2}^{k+2} \in A^{2}\left(\Omega_{m}\right) .
$$

Hence, the range of the operator $T_{\phi}$ contains all the monomials of the form $z_{1}^{k+2} z_{2}^{k+2}$, and so the range of $T_{\phi}$ is infinite-dimensional.

If $g\left(z_{1}, z_{2}\right) \in A^{2}\left(\Omega_{m}\right)$, then its series expansion will be

$$
g\left(z_{1}, z_{2}\right)=\sum_{\alpha_{1}=0}^{\infty} \sum_{\alpha_{2}=\alpha_{1}}^{\alpha_{1}+r-1} g_{\alpha_{1} \alpha_{2}} \frac{z_{2}^{\alpha_{2}} z_{1}^{\alpha_{1}}}{c_{\left(\alpha_{1}, \alpha_{2}\right)}}=\sum_{\alpha_{1}=0}^{\infty} \sum_{\alpha_{2}=\alpha_{1}}^{\alpha_{1}+r-1}\left\langle g(z), \frac{z^{\alpha}}{c_{\alpha}}\right\rangle \frac{z^{\alpha}}{c_{\alpha}},
$$

where

$$
r= \begin{cases}m / 2 & \text { if } m \text { is even, } \\ (m-1) / 2 & \text { if } m \text { is odd }\end{cases}
$$

The norm of $g\left(z_{1}, z_{2}\right)$ is given by

$$
\|g\|_{A^{2}\left(\Omega_{m}\right)}^{2}=\sum_{\alpha_{1}=0}^{\infty} \sum_{\alpha_{2}=\alpha_{1}}^{\alpha_{1}+r-1}\left|g_{\alpha_{1} \alpha_{2}}\right|^{2},
$$


and the norm of $T_{\phi}(g)$ is

$$
\begin{aligned}
\left\|T_{\phi}(g)\right\|_{A^{2}\left(\Omega_{m}\right)}^{2} & =\left\|\sum_{\alpha_{1}=0}^{\infty} \sum_{\alpha_{2}=\alpha_{1}}^{\alpha_{1}+r-1}\left\langle\frac{z_{1}}{\bar{z}_{1}} \cdot g(z), \frac{z^{\alpha}}{c_{\alpha}}\right\rangle \frac{z^{\alpha}}{c_{\alpha}}\right\|^{2} \\
& =\sum_{\alpha_{1}=0}^{\infty} \sum_{\alpha_{2}=\alpha_{1}}^{\alpha_{1}+r-1}\left|\left\langle\frac{z_{1}}{\bar{z}_{1}} \cdot g(z), \frac{z^{\alpha}}{c_{\alpha}}\right\rangle\right|^{2} \\
& =\sum_{\alpha_{1}=0}^{\infty} \sum_{\alpha_{2}=\alpha_{1}}^{\alpha_{1}+r-1}\left|\left\langle\frac{z_{1}}{\bar{z}_{1}} \cdot \sum_{\beta} g_{\beta} \frac{z^{\beta}}{c_{\beta}}, \frac{z^{\alpha}}{c_{\alpha}}\right\rangle\right|^{2} \\
& =\sum_{\alpha_{1}=2}^{\infty} \sum_{\alpha_{2}=\alpha_{1}}^{\alpha_{1}+r-1}\left|\left\langle\frac{z_{1}}{\bar{z}_{1}} \cdot g_{\left(\alpha_{1}-2, \alpha_{2}\right)} z_{1}^{\alpha_{1}-2} z_{2}^{\alpha_{2}}, \frac{z^{\alpha}}{c_{\alpha}}\right\rangle\right|^{2}
\end{aligned}
$$

by orthogonality of monomials

$$
=\sum_{\alpha_{1}=2}^{\infty} \sum_{\alpha_{2}=\alpha_{1}}^{\alpha_{1}+r-1}\left|g_{\left(\alpha_{1}-2, \alpha_{2}\right)} \frac{c_{\left(\alpha_{1}-1, \alpha_{2}\right)}}{c_{\alpha}}\right|^{2}
$$

then we shift the indices

$$
=\sum_{\alpha_{1}=0}^{\infty} \sum_{\alpha_{2}=\alpha_{1}}^{\alpha_{1}+r-1}\left|\widetilde{g}_{\alpha_{1} \alpha_{2}}\right|^{2}
$$

where

$$
\widetilde{g}_{\alpha_{1} \alpha_{2}}= \begin{cases}0 & \text { if } \alpha_{1}=\alpha_{2} \text { or } \alpha_{1}=\alpha_{2}+1, \\ \left(c_{\left(\alpha_{1}+1, \alpha_{2}\right)} / c_{\left(\alpha_{1}+2, \alpha_{2}\right)}\right) g_{\alpha_{1} \alpha_{2}} & \text { otherwise. }\end{cases}
$$

The ratio $c_{\left(\alpha_{1}+1, \alpha_{2}\right)}^{2} / c_{\left(\alpha_{1}+2, \alpha_{2}\right)}^{2}$ is uniformly bounded by a constant. Indeed, each integral on $X$ and $Y_{m}$ has a uniform bound from above (say $C_{X}$ and $C_{Y_{m}}$ ) because of conditions (2.1) and (2.2). Furthermore, we compute the integrals on the polydisc $Z$ explicitly and estimate as follows:

$$
\begin{aligned}
\frac{c_{\left(\alpha_{1}+1, \alpha_{2}\right)}^{2}}{c_{\left(\alpha_{1}+2, \alpha_{2}\right)}^{2}} & \leq \frac{C_{X}+C_{Y_{m}}+\pi\left(e^{2 \alpha_{1}+4}\right) /\left(\alpha_{1}+2\right) \cdot \pi\left(2^{2 \alpha_{2}+2}\right) /\left(\alpha_{2}+1\right)}{\pi\left(e^{2 \alpha_{1}+2}\right) /\left(\alpha_{1}+1\right) \cdot \pi\left(2^{2 \alpha_{2}+2}\right) /\left(\alpha_{2}+1\right)} \\
& \leq \frac{C_{X}+C_{Y_{m}}}{\pi^{2}}+e^{2}=C .
\end{aligned}
$$


This estimate implies

$$
\left|\widetilde{g}_{\alpha_{1} \alpha_{2}}\right|^{2} \leq C \cdot\left|g_{\alpha_{1} \alpha_{2}}\right|^{2} \text {, for all }\left(\alpha_{1}, \alpha_{2}\right) \in R_{m} .
$$

Thus, from equations (2.4), (2.5) and (2.9), it follows that

$$
\left\|T_{\phi}(g)\right\|_{A^{2}\left(\Omega_{m}\right)}^{2} \leq C \cdot\|g\|_{A^{2}\left(\Omega_{m}\right)}^{2} .
$$

Finally, we calculate the powers of $T_{\phi}$ :

$$
\begin{aligned}
T_{\phi}^{2}\left(z^{\alpha}\right) & =T_{\phi} \cdot T_{\phi}\left(z^{\alpha}\right)=T_{\phi}\left(\frac{c_{\left(\alpha_{1}+1, \alpha_{2}\right)}^{2}}{c_{\left(\alpha_{1}+2, \alpha_{2}\right)}^{2}} z_{1}^{\alpha_{1}+2} z_{2}^{\alpha_{2}}\right) \\
& =\frac{c_{\left(\alpha_{1}+1, \alpha_{2}\right)}^{2}}{c_{\left(\alpha_{1}+2, \alpha_{2}\right)}^{2}} \cdot \frac{c_{\left(\alpha_{1}+3, \alpha_{2}\right)}^{2}}{c_{\left(\alpha_{1}+4, \alpha_{2}\right)}^{2}} z_{1}^{\alpha_{1}+4} z_{2}^{\alpha_{2}} .
\end{aligned}
$$

As for the third power,

$$
T_{\phi}^{3}\left(z^{\alpha}\right)=\frac{c_{\left(\alpha_{1}+1, \alpha_{2}\right)}^{2}}{c_{\left(\alpha_{1}+2, \alpha_{2}\right)}^{2}} \cdot \frac{c_{\left(\alpha_{1}+3, \alpha_{2}\right)}^{2}}{c_{\left(\alpha_{1}+4, \alpha_{2}\right)}^{2}} \cdot \frac{c_{\left(\alpha_{1}+5, \alpha_{2}\right)}^{2}}{c_{\left(\alpha_{1}+6, \alpha_{2}\right)}^{2}} z_{1}^{\alpha_{1}+6} z_{2}^{\alpha_{2}} .
$$

Continuing in this fashion, the $k$ th power of the operator is:

$$
T_{\phi}^{k}\left(z^{\alpha}\right)=\frac{c_{\left(\alpha_{1}+1, \alpha_{2}\right)}^{2}}{c_{\left(\alpha_{1}+2, \alpha_{2}\right)}^{2}} \cdot \frac{c_{\left(\alpha_{1}+3, \alpha_{2}\right)}^{2}}{c_{\left(\alpha_{1}+4, \alpha_{2}\right)}^{2}} \cdots \frac{c_{\left(\alpha_{1}+2 k-1, \alpha_{2}\right)}^{2}}{c_{\left(\alpha_{1}+2 k, \alpha_{2}\right)}^{2}} z_{1}^{\alpha_{1}+2 k} z_{2}^{\alpha_{2}} .
$$

In equation (2.12), if $2 k<r$, then there exists an $\left(\alpha_{1}, \alpha_{2}\right) \in R_{m}$ such that $\left(\alpha_{1}+2 k, \alpha_{2}\right) \in R_{m}$, see the discussion in Remark 2.4, so $z_{1}^{\alpha_{1}+2 k} z_{2}^{\alpha_{2}} \in A^{2}\left(\Omega_{m}\right)$ and $T_{\phi}^{k} \not \equiv 0$ on $A^{2}\left(\Omega_{m}\right)$.

However, in equation (2.12), if $2 k \geq r$, then for all $\left(\alpha_{1}, \alpha_{2}\right) \in R_{m}$, we have $\left(\alpha_{1}+2 k, \alpha_{2}\right) \notin R_{m}$ by Remark 2.4 , so we see that $z_{1}^{\alpha_{1}+2 k} z_{2}^{\alpha_{2}} \notin$ $A^{2}\left(\Omega_{m}\right)$ and $T_{\phi}^{k} \equiv 0$ on $A^{2}\left(\Omega_{m}\right)$, that is, $T_{\phi}$ is a nilpotent operator of degree $k$ on $A^{2}\left(\Omega_{m}\right)$.

We illustrate the main arguments of the proof in the following example.

Example 2.5. Set $m=9$. Then the monomial $z_{1}^{\alpha_{1}} z_{2}^{\alpha_{2}}$ is in $A^{2}\left(\Omega_{9}\right)$ if and only if $\alpha_{1}+4>\alpha_{2} \geq \alpha_{1}$. The exponents of the monomial in $A^{2}\left(\Omega_{9}\right)$ are marked on the lattice in Figure 2. It can be noted that $T_{\phi}$ acts like a shift on the lattice; it takes $\left(\alpha_{1}, \alpha_{2}+2\right)$ to $\left(\alpha_{1}+2, \alpha_{2}+2\right)$. Thus, if $T_{\phi}$ is applied on any monomial two times, then the exponent 


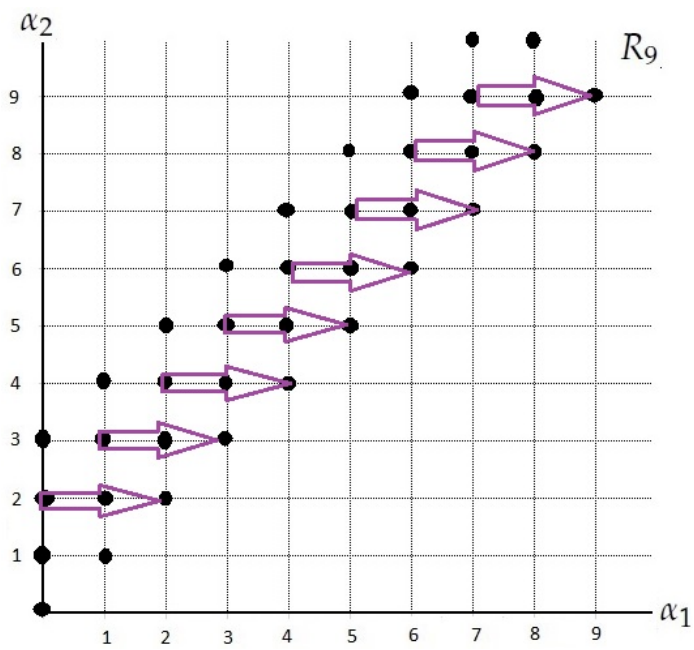

FIGURE 2. Representation of the lattice $R_{m}$ for $m=9$ and the action of $T_{\phi}$ on $R_{m}$.

of the monomial runs out of the lattice $R_{9}$, that is, if $z_{1}^{\alpha_{1}} z_{2}^{\alpha_{2}} \in A^{2}\left(\Omega_{9}\right)$, then

$$
T_{\phi} \cdot T_{\phi}\left(z_{1}^{\alpha_{1}} z_{2}^{\alpha_{2}}\right)=\frac{c_{\left(\alpha_{1}+1, \alpha_{2}\right)}^{2}}{c_{\left(\alpha_{1}+2, \alpha_{2}\right)}^{2}} \cdot \frac{c_{\left(\alpha_{1}+3, \alpha_{2}\right)}^{2}}{c_{\left(\alpha_{1}+4, \alpha_{2}\right)}^{2}} z_{1}^{\alpha_{1}+4} z_{2}^{\alpha_{2}} \notin A^{2}\left(\Omega_{9}\right),
$$

and so $T_{\phi}^{2} \equiv 0$ on $A^{2}\left(\Omega_{9}\right)$.

Acknowledgments. We thank the referees for constructive comments on Proposition 2.2 and also for the useful editorial remarks on the exposition of the article.

\section{REFERENCES}

1. Patrick Ahern and Željko Čučković, Products of Toeplitz operators on the Bergman space, Illinois J. Math. 45 (2001), 113-121.

2. A A theorem of Brown-Halmos type for Bergman space Toeplitz operators, J. Funct. Anal. 187 (2001), 200-210.

3. Alexandru Aleman and Dragan Vukotić, Zero products of Toeplitz operators, Duke Math. J. 148 (2009), 373-403. 
4. Wolfram Bauer and Trieu Le, Algebraic properties and the finite rank problem for Toeplitz operators on the Segal-Bargmann space, J. Funct. Anal. 261 (2011), 2617-2640.

5. Arlen Brown and P.R. Halmos, Algebraic properties of Toeplitz operators, J. reine angew. Math. 213 (1963/1964), 89-102.

6. Boo Rim Choe and Hyungwoon Koo, Zero products of Toeplitz operators with harmonic symbols, J. Funct. Anal. 233 (2006), 307-334.

7. Boo Rim Choe, Hyungwoon Koo and Young Joo Lee, Zero products of Toeplitz operators with $n$-harmonic symbols, Int. Equat. Oper. Theor. 57 (2007), 43-66.

8. Boo Rim Choe, Young Joo Lee, Kyesook Nam and Dechao Zheng, Products of Bergman space Toeplitz operators on the polydisk, Math. Ann. 337 (2007), 295-316.

9. Caixing $\mathrm{Gu}$, Products of several Toeplitz operators, J. Funct. Anal. 171 (2000), 483-527.

10. Kun Yu Guo, A problem on products of Toeplitz operators, Proc. Amer. Math. Soc. 124 (1996), 869-871.

11. Steven G. Krantz, Function theory of several complex variables, AMS Chelsea Publishing, Providence, RI, 2001.

12. Jan J.O.O. Wiegerinck, Domains with finite-dimensional Bergman space, Math. Z. 187 (1984), 559-562.

Department of Mathematics, Texas A\&M University-Commerce, Commerce, TX 75429

Email address: mehmet.celik@tamuc.edu

University of Michigan, Department of Mathematics and Statistics, DearBORN, MI 48128

Email address: zeytuncu@umich.edu 